\title{
Ain-Shams experience in the management of end stage fecal incontinence using antegrade continence enema
}

\author{
Ahmed Alaa, FRCS(Ed) \\ Department of General Surgery, Ain-Shams University, Cairo, Egypt
}

(Presented in the 9th annual conference of the Egyptian Group of Colon \& Rectal Surgeons held in July 2007 in Sharm El Sheikh).

\begin{abstract}
The Malone antegrade continence enema (ACE) has revolutionized the management of intractable fecal incontinence. In this technique, the appendix or neoappendix is brought to the abdominal wall creating a small stoma. Thereby, antegrade enemas can be administered to empty the colorectum and prevent accidents of fecal incontinence. The aim of this study was to assess the results of the ACE in adults suffering from end-stage fecal incontinence. Ten adult male patients with severe incontinence due to different etiologies were included in the study. The appendix was used in eight and Ileal- neoappendix was created in two.

The ideal appendicostomy was that anastomosed to V-skin flap with an antireflux procedure. All the patients achieved almost complete cleanliness with those having neurogenic cause for their incontinence being the most satisfied regardless the stoma complication or appearance.

The impact on social activities and quality of life caused by bowel dysfunction was reduced in all ten patients.

We conclude that the ACE technique can be reasonably used to treat selected adult patients suffering from fecal incontinence after all other measures have failed or are not applicable due to the expense or the complex technological back up needed to maintain their success.
\end{abstract}

\section{Introduction:}

In 1990, Malone et al. ${ }^{1}$ described the antegrade continence enema (ACE) technique, creating a continent stoma by implanting the tip of the appendix in a non-refluxing manner into the cecum and bringing the base out onto the abdominal wall. Through this small appendicostomy, antegrade enemas could be administered to empty the colorectum and prevent fecal incontinence or constipation. In several studies the ACE has been safe and effective among children suffering from fecal incontinence or chronic constipation due to spina bifida or anorectal malformations. ${ }^{2-5}$ Later reports have indicated that reimplantation of the appendix to avoid fecal leakage is unnecessary and most authors simply bring out the tip of the appendix ${ }^{2-4,6}$ the technique has been further modified by creating a "neoappendix" from ileum or colon as an alternative in patients without an appendix. ${ }^{7-12}$

Although many studies ${ }^{13-19}$ have shown that the procedure could relieve symptoms in adult patients suffering from fecal incontinence or chronic constipation due to myriads of acquired, congenital, neurologic or anatomic defects, there is yet no single Egyptian study, to the best of our knowledge, that has addressed this issue.

This report describes the outcome of this novel technique in adult patients with end stage fecal incontinence in Ain Shams University Hospitals.

\section{Patients and methods:}

Between Dec.2004-Dec.2006, ten dexterous male patients with end stage fecal incontinence with mean age of $19 \mathrm{yrs}$ (range 18-24) underwent antegrade continence enema (ACE) with a mean follow up of 12 months (range 9-30).

\section{Surgical procedures:}

The patients underwent standard bowel preparation; antibiotics were given with the 
induction of general anesthesia with the patient in the supine position. Transverse celiotomy in the right lower abdomen was used and the approximate site of the stoma was marked Figure(1).

The appendix was identified, its tip excised and a Nelaton (Fr10) catheter was lubricated and gently introduced into the lumen and maneuvered into the cecum to verify patency. A window was opened in a bloodless fold in mesoappendix next to the base of the appendix preserving the artery Figure(2A). While the catheter is inserted in place, an anti-reflux purse string Vicryl 3/0 stitch was taken in the cecum through the created window in the mesoappendix Figure(2B).

The tip of the appendix was then delivered through the right iliac fossa or periumbilically if the topography of the patient does not allow for the former positioning of the appendicostomy.

Then a small stoma was created from the appendix directly or using a V-Y plasty in the skin after fish tailing the tip of the appendix Figure(3).

In patients without an appendix or if the appendix was not suitable for the procedure, a neoappendix was constructed from the terminal ileum. The distal few $\mathrm{cms}$ in continuity with the cecum was transected and delivered through the skin as a stoma. The continuity of the intestinal tract was accomplished by anastomosing the proximal ileum to the cecum or ideally to transverse colon Figure(4).

Following both procedures, a Nelaton catheter (Fr10) was left in situ for 2 weeks.

Patients were asked to compare bowel function before the operation and at followup, using a scale in which 0 was worst possible and 100 ideal.

\section{Postoperative strategy:}

Irrigations started as soon as patients are eating in the early postoperative period taking advantage of gastrocolic reflex.

Irrigations consisted initially of 100-200 $\mathrm{mL}$ of tap water, with $50-100 \mathrm{~mL}$ increments every 2 to 3 days. The water was injected into the catheter using a $50 \mathrm{ml}$ syringe with a nozzle. The patient monitored continence, since trial and error (with respect to volume and frequency) determined what works best for each patient. They were instructed to catheterize the stoma at least once daily to prevent its stricture.

\section{Results:}

Ten male patients with end stage fecal incontinence were included in the present series. The etiologies of their defecatory disorder are shown in Table(1). Operative procedures and postoperative results are summarized in Table(2).

The mean score of bowel function before the operation was 14(0-30).

Table (1): Patients included in the study.

\begin{tabular}{|l|c|}
\hline Cause of symptoms & No. of patients \\
\hline Neurologic (spina bifida) & 5 \\
\hline Irreparable sphincter injury & 3 \\
\hline After operation for Hirschsprung & 2 \\
\hline Total & 10 \\
\hline
\end{tabular}




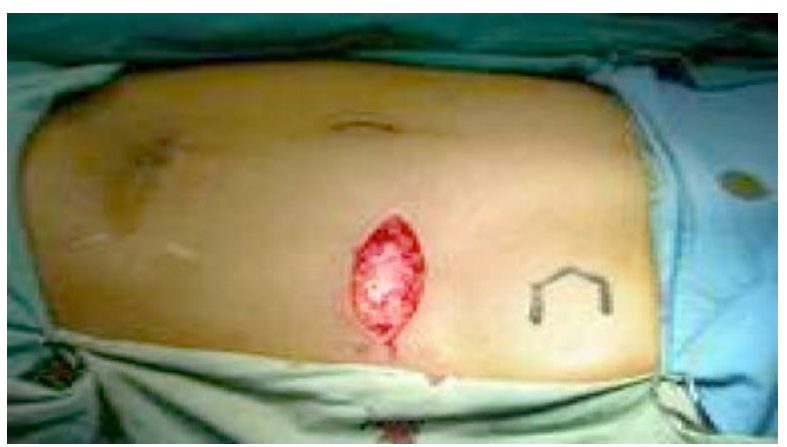

Figure (1): Transverse celiotomy with marking of the skin flap.

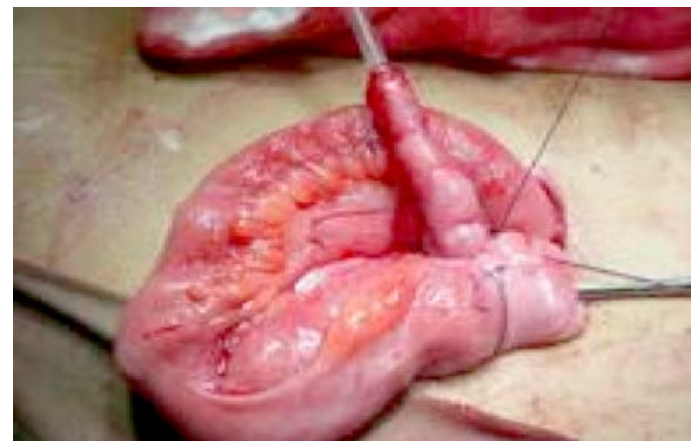

A

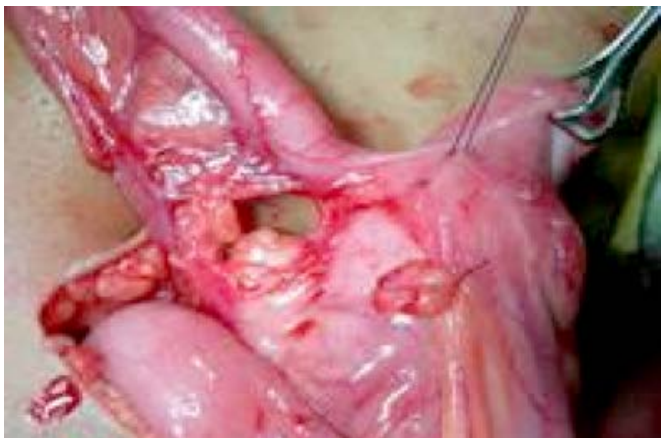

$B$

Figure (2): Creation of a window in the mesoappendix near the base of the appendix (A) with the purse string antireflux stitch inserted while a t $10 \mathrm{Fr}$ catheter is in- situ (B).

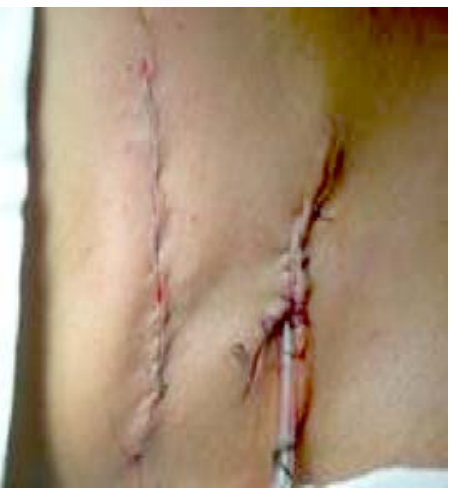

A

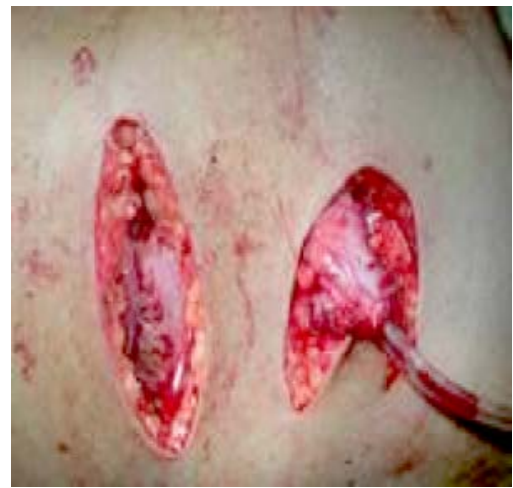

$B$

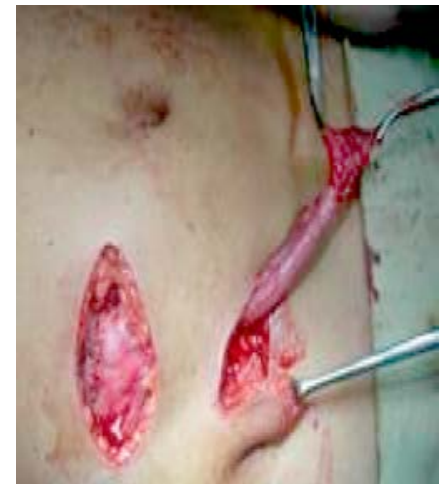

C

Figure (3): The skin flap is created and the tip of the appendix is fishtailed (A) with the tip of the skin flap inset to the fishtail (B) and finally the skin edges are approximated as in a V-Y flap $(C)$.

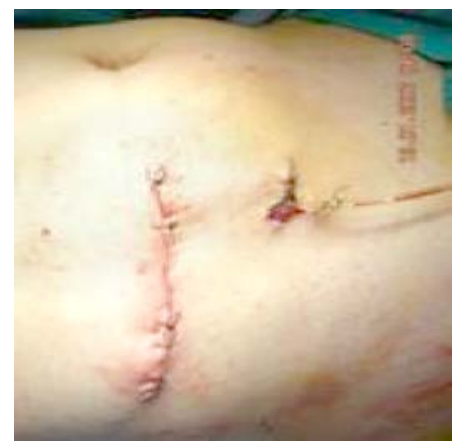

A

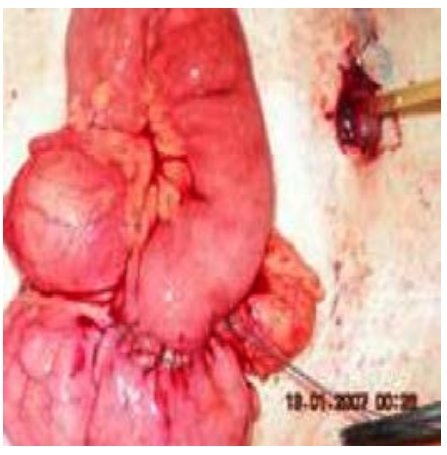

$B$

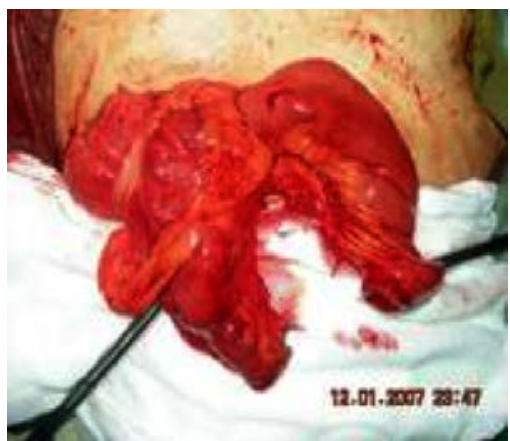

$C$

Figure (4): Ileal neoappendix: The terminal ileum is divided few centimeters from the ileocecal valve $(A)$, the remainiing ileum is anastomosed in this patient to the cecum $(B)$ with the short ileal loop brought to the skin and fashioned as a stoma $(C)$. 
Table (2): Operative procedures and postoperative results.

\begin{tabular}{|c|c|c|c|c|c|c|c|c|}
\hline no. & $\begin{array}{c}\text { Etiology } \\
\text { of FI }\end{array}$ & Procedure & $\begin{array}{c}\text { Superficial } \\
\text { infection }\end{array}$ & $\begin{array}{c}\text { Deep } \\
\text { infection }\end{array}$ & \begin{tabular}{|c|}
$\begin{array}{c}\text { Reflux } \\
\text { inbetween } \\
\text { washes }\end{array}$ \\
\end{tabular} & Spotting & Stenosis & $\begin{array}{c}\text { Functional score } \\
\text { preop....... postop. }\end{array}$ \\
\hline 1 & $\begin{array}{l}\text { Spina } \\
\text { bifida }\end{array}$ & $\begin{array}{l}\text { Appendico- } \\
\text { stomy } \\
\text { without } \\
\text { antireflux }\end{array}$ & - & $+\mathrm{ve}$ & ++ & $+\mathrm{ve}$ & $y($ redo $)$ & $5 \ldots \ldots \ldots \ldots$ \\
\hline 2 & $\begin{array}{l}\text { Spina } \\
\text { bifida }\end{array}$ & $\begin{array}{l}\text { Appendico- } \\
\text { stomy } \\
\text { without } \\
\text { antireflux }\end{array}$ & $+\mathrm{ve}$ & - & ++ & $+\mathrm{ve}$ & $y($ redo $)$ & $10 \ldots \ldots \ldots . . . . .100$ \\
\hline 3 & $\begin{array}{l}\text { Spina } \\
\text { bifida }\end{array}$ & $\begin{array}{l}\text { Appendico- } \\
\text { stomy } \\
\text { without } \\
\text { antireflux }\end{array}$ & +ve & - & ++ & $+\mathrm{ve}$ & $\mathrm{y}($ dilated $)$ & $10 \ldots \ldots \ldots . . . . . . .100$ \\
\hline 4 & $\begin{array}{l}\text { Spina } \\
\text { bifida }\end{array}$ & $\begin{array}{l}\text { Appendico- } \\
\text { stomy with } \\
\text { V-Y plasty } \\
\text { \&antireflux }\end{array}$ & - & - & - & - & - & 15 ............. 100 \\
\hline 5 & $\begin{array}{l}\text { Spina } \\
\text { bifida }\end{array}$ & $\begin{array}{l}\text { Appendico- } \\
\text { stomy with } \\
\text { antireflux }\end{array}$ & +ve & - & + & $+\mathrm{ve}$ & $\mathrm{y}($ dilated $)$ & $20 \ldots \ldots \ldots . . . . .100$ \\
\hline 6 & $\begin{array}{l}\text { Irreparable } \\
\text { Sphincter } \\
\text { Injury }\end{array}$ & $\begin{array}{l}\text { Appendico- } \\
\text { stomy with } \\
\text { antireflux }\end{array}$ & $+\mathrm{ve}$ & - & + & - & - & $0 \ldots \ldots \ldots$ \\
\hline 7 & $\begin{array}{l}\text { Irreparable } \\
\text { Sphincter } \\
\text { Injury }\end{array}$ & $\begin{array}{l}\text { Appendico- } \\
\text { stomy with } \\
\text { V-Y plasty } \\
\text { \&antireflux } \\
\end{array}$ & - & - & - & - & - & $15 \ldots \ldots \ldots . . . . .80$ \\
\hline 8 & $\begin{array}{l}\text { Irreparable } \\
\text { Sphincter } \\
\text { Injury }\end{array}$ & $\begin{array}{l}\text { Appendico- } \\
\text { stomy with } \\
\text { V-Y plasty } \\
\text { \&antireflux }\end{array}$ & - & - & - & - & - & 10 .............. 100 \\
\hline 9 & $\begin{array}{l}\text { After } \\
\text { operation for } \\
\text { Hirschsprung }\end{array}$ & $\begin{array}{l}\text { Ileal- } \\
\text { neoappendix }\end{array}$ & - & - & $\begin{array}{l}+++ \\
\text { (redo) }\end{array}$ & $+\mathrm{ve}$ & - & $30 \ldots \ldots \ldots \ldots . . .65$ \\
\hline 10 & $\begin{array}{l}\text { After } \\
\text { operation for } \\
\text { Hirschsprung }\end{array}$ & $\begin{array}{l}\text { Ileal- } \\
\text { neoappendix }\end{array}$ & - & - & - & $+\mathrm{ve}$ & - & $25 \ldots \ldots \ldots . . . .60$ \\
\hline
\end{tabular}


The appendix was used orthotopically in eight patients without antireflux in three, with antireflux in two and with V-Y inlays skin flap tunnel and antireflux in the last three patients.

Non-tubularized Ileal neoappendix was created in two patients. The proximal ileum was anastomosed to the cecum $(n=1)$ or transverse colon $(\mathrm{n}=1)$.

The stoma was placed in right iliac fossa in nine patients and periumbilically in one patient.

\section{Complications:}

Four wounds surrounding the stoma were superficially infected and responded to conservative measures.

Deep infection occurred in one patient with appendicostomy.

Skin -level stomal stenosis affected four appendicostomies including the previous patient with deep infection. The stoma was dilated in two and revised in the other two patients.

Reflux after wash was present in five patients as follows:

- Four minimal reflux, (3\&1 appendicostomy without \& with antireflux, respectively)

- One major reflux in a patient with ileal neoappendix in whom the proximal ileum was anastomosed to the cecum. Redo ileotransverse anastomosis abolished the reflux.

Reflux between wash and/or mucus spotting affected seven patients as follows:

- Three refluxed air \& stool. They were those with appendicostomy without antireflux stitch. The patients were able to deal with the reflux and needed nothing more.

- Two with appendicostomy plus antireflux, refluxed air only in addition to minimum spotting from the exposed appendicial mucosa.

- One troublesome reflux and spotting from exposed mucosa (the patient with ileal neoappendix where the ileum was connected to the cecum. The reflux was controlled after the redo).

- One with spotting from exposed ileal mucosa but with no reflux and the patient did not find the spotting cumbersome.

\section{Postoperative continence:}

No accidents of incontinence and complete dryness were achieved in six patients (5 with neurologic etiology and 1 with sphincter injury).

Once or twice mishap of incontinence every month affected two patients with sphincter injury.

Incontinence once or more every week was found in the last two patients after an operation for Hirschsprung.

The postoperative functional score was 89(60-100).

\section{Use of ACE for irrigation:}

\section{1- Frequency:}

Once every day in five patients (4-Spina bifida, 1-sphincter injury).

Once every second day in two patients (1 Spina bifida, 1-sphincter injury).

Twice every day in three patients (1- sphincter injury, 2- post Hirschsprung operation). 2- Volume:

Volume of water used for the enema was 150-1000 ml (mean 250). The volume again was greatest in patients with post Hirschsprung incontinence. The volume with time was in a decreasing order.

Time taken to initiate defecation after the enema was 10-50 minutes (mean 20); the longest was in post Hirschsprung patients.

\section{Satisfaction:}

Overall satisfaction was very high in the five patients with spina bifida, high in the three patients with sphincter injury. The least satisfied were the patients with previous operation for Hirschsprung.

\section{Discussion:}

The concept of the antegrade continence (or colonic) enema (ACE) originated from Malone et al. ${ }^{1}$ in 1990 . It had been previously demonstrated ${ }^{20}$ that antegrade colonic irrigation via a rectal tube dramatically decreased fecal soiling in children with spina bifida. Malone expanded this technique by using the appendix as a conduit for the instillation of colonic irrigant in a way similar to Mitrofanoff ${ }^{21}$ technique for intermittent urinary catheterization via a continent channel.

The theory of the ACE is that patients may 
become clean when managed with routine large volume antegrade enemas that cause complete colonic evacuation

The ACE procedure has revolutionized the lives of many patients with intractable constipation or incontinence particularly in the pediatric age group. Because of this success, Wheeler and Malone 22 in 1991 have advocated a ban on routine appendectomy without evidence of a pathological condition.

This study shows that Malone antegrade continence enema (ACE) is safe and effective treatment for selected adult patients suffering from severe fecal incontinence.

Overall, the ACE was successful in all the patients in over a mean follow up of 12 months. In comparison to previous reports $13,15,18,19$ in the adult incontinent population, a similar perioperative complication profile was achieved and an improved long-term functional outcome was observed.

These preliminary results indicate that the ACE can relieve colorectal problems in patients with neurological lesions who often suffer from severe constipation or fecal incontinence as has been demonstrated in other studies. ${ }^{16}$, 17, 23 It is interesting to note that this group reported the highest satisfaction, though the stoma constructed in four of these patients was not the friendly invisible one. To the contrary, repeated dilatation was done for two and surgical redo was needed for the other two stomas. This very high satisfaction despite the complicated stoma may be explained by the fact that these patients have never "tasted" a normal bowel habit.

Furthermore, the results in patients with incontinence due to severe anal sphincter lesions are comparable to results from the creation of a neosphincter from the Gracilis muscle $24-26$ or the implantation of artificial anal sphincter $26-28$ that are far more demanding procedures both technically and financially. Therefore, the ACE technique, as a simpler and smaller operation, may be an alternative to these more extensive procedures. However, the satisfaction of the patients with the sphincter injury was not as high as anticipated, at least by the surgeon, despite the excellent functional score and the merely visible non-refluxing stoma in two of these patients. This paradox largely reflects the depression associated with the devastating incontinence in a previously normal person particularly when the perfect past is compared with the unpleasant present!

The "congenital group" in the present study was the least satisfied and reported the lowest functional score in comparison with the previous groups. However, neither of the two patients in the group regretted the operation and they are enjoying a much better life with less social restrictions and embarrassment. The inherent intestinal neuronal dysplasia affecting the patients with Hirschsprung could participate in the uncontrolled imperfect response to the antegrade enema. ${ }^{29,} 30$

As in other studies, ${ }^{2-4,6}$ the present results indicate that reimplanting the appendix is unnecessary. The tip of the appendix was simply brought out. There is a high incidence of morbidity (mucus discharge, stenosis) related to skin leveling of the appendix or neoappendix. An inlay of skin at the cutaneous stomal anastomosis, as applied in the last three appendicostomies in this report, is not only associated with less stenosis but also avoids problems with exposed mucosa and has an overall better cosmetic effect.

Our result using this technique is in accordance with other series using the same principles when creating the stoma. 2,31

If the appendix is not available or insufficient, a number of other techniques have been used to create a substitute for the appendix. ${ }^{7-12}$ In the present study, and unlike others, ${ }^{7,8}$ we did not reduce the caliber of the terminal ileum in an attempt to avoid the reported complications of added staples or suture line and the reported long-term increased difficulty in catheterization due to stomal stenosis. However, this compromise was on the expense of providing a non-visible nonspotting stoma.

The reliance on the ileocecal valve to prevent cecal contents from refluxing in to the ileal conduit led to massive reflux in the patient in whom the proximal ileum was anastomosed to the cecum. This patient needed revision with an ileotransverse anastomosis, which abolished the reflux and was consequently applied in the other patient with neoappendicostomy in this work. 
For the colonic irrigation, tap water is used with the policy of gradual increase in the amount of water to prevent the abdominal discomfort experienced by some patients during the wash-outs. ${ }^{15-18}$ So far, this sliding scale strategy has worked well with our patients. Close follow-up was then maintained to readjust the volume and the frequency of $\mathrm{ACE}$ irrigation until the patient's objective report of continence and regular defecation. It is in essence a matter of trial and error and each patient will independently pick up what serves him best. There was continuous emphasis on catheterization of the stoma at least once daily in case no irrigation is needed especially in the early postoperative period as the stomas close quickly if catheterizations are not performed on schedule.

Thus, it is most important that all patients are well motivated, and understand that some time is necessary to tailor the procedure to his or her needs. Therefore, we did not perform this procedure unless the patients and their caregivers had shown good compliance with our other instructions.

The present study confirms that the treatment with antegrade continence enemas in adults is well tolerated with only transient side effects. Since this is a minor and reversible operation, it could often be indicated in selected patients with severe defecatory disorders resistant to other therapies. However, larger studies are needed for further evaluation, and long-term results have yet to be described.

\section{Conclusion:}

ACE is not a panacea for end-stage fecal incontinence. It is, nevertheless, a middle ground solution between a helpless past, an expensive technically demanding present. Yet it is preserving the "status quo" sphincter anatomy for future Malone's style ingenious innovations.

\section{Acknowledgment:}

The credit of this work goes to Dr. Ahmed Abdul Aziz Abou Zeid, professor of general surgery in Ain-Shams University. He is first to introduce this novel technique in adults to our institution if not in the whole country. This is in addition to his active participation and assistance in most of the procedures performed in this series.

\section{References:}

1- Malone PS, Ransley PG, Kiely EM: Preliminary report: the antegrade continence enema. Lancet 1990; 336(8725): 1217-8.

2- Griffiths DM, Malone PS: The Malone antegrade continence enema. $J$ Pediatr Surg 1995; 30(1): 68-71.

3- Squire R, Kiely EM, Carr B, Ransley PG, Duffy PG: The clinical application of the Malone antegrade colonic enema. J Pediatr Surg 1993; 28(8): 1012-5.

4- Koyle MA, Kaji DM, Duque M, Wild J, Galansky SH: The Malone antegrade continence enema for neurogenic and structural fecal incontinence and constipation. J Urol 1995; 154(2 Pt 2): 759-61.

5- Soylet Y, Yesildag E, Besik C, Emir H: Antegrade continence enema--an analysis of 20 children with faecal incontinence. Eur J Pediatr Surg 2006; 16(4): 251-4.

6- Hill J, Stott S, MacLennan I: Antegrade enemas for the treatment of severe idiopathic constipation. Br J Surg 1994; 81(10): 1490-1.

7- Christensen P, Buntzen S, Krogh K, Laurberg S: Ileal neoappendicostomy for antegrade colonic irrigation. Br J Surg 2001; 88(12): 1637-8.

8- Tackett LD, Minevich E, Benedict JF, Wacksman J, Sheldon CA: Appendiceal versus ileal segment for antegrade continence enema. J Urol 2002; 167(2): 683-6.

9- Herndon CD, Cain MP, Casale AJ, Rink RC: The colon flap/extension Malone antegrade continence enema: an alternative to the Monti-Malone antegrade continence enema. J Urol 2005; 174(1): 299-302.

10-Ahn SM, Han SW, Choi SH: The results of antegrade continence enema using a retubularized sigmoidostomy. Pediatr Surg Int 2004; 20(7): 488-91.

11-Kurzrock EA, Karpman E, Stone AR: Colonic tubes for the antegrade continence enema: comparison of surgical technique. J Urol 2004; 172(2): 700-2.

12-Churchill BM, De Ugarte DA, Atkinson JB: Left-colon antegrade continence enema 
(LACE) procedure for fecal incontinence. J Pediatr Surg 2003; 38(12): 1778-80.

13-Poirier M, Abcarian H, Nelson R: Malone antegrade continent enema: an alternative to resection in severe defecation disorders. Dis Colon Rectum 2007; 50(1): 22-8.

14-Portier G, Ghouti L, Kirzin S, Chauffour M, Lazorthes F: Malone antegrade colonic irrigation: ileal neoappendicostomy is the preferred procedure in adults. Int $J$ Colorectal Dis 2006; 21(5): 458-60.

15-Lefevre JH, Parc Y, Giraudo G, Bell S, Parc R, Tiret E: Outcome of antegrade continence enema procedures for faecal incontinence in adults. Br J Surg 2006; 93(10): 1265-9.

16-Teichman JM, Zabihi N, Kraus SR, Harris JM, Barber DB: Long-term results for Malone antegrade continence enema for adults with neurogenic bowel disease. Urology 2003; 61(3): 502-6.

17-Teichman JM, Harris JM, Currie DM, Barber DB: Antegrade continence enema for adult neurogenic patients. Can J Urol 1998; 5(3): 603-606.

18-Moore EM: Malone antegrade continence enema for faecal incontinence and constipation in adults. Br J Surg 1998; 85(11): 1594.

19-Gerharz EW, Vik V, Webb G, Leaver R, Shah PJ, Woodhouse CR: The value of the MACE (Malone antegrade colonic enema) procedure in adult patients. J Am Coll Surg 1997; 185(6): 544-7.

20-Shandling B, Gilmour RF: The enema continence catheter in spina bifida: successful bowel management. J Pediatr Surg 1987; 22(3): 271-3.

21-Mitrofanoff P: Trans-appendicular continent cystostomy in the management of the neurogenic bladder. Chir Pediatr 1980; 21(4): 297-305.

22-Wheeler RA, Malone PS: Use of the appendix in reconstructive surgery: a case against incidental appendicectomy. $\mathrm{Br} \mathrm{J}$ Surg 1991; 78(11): 1283-5.

23-Lemelle JL, Guillemin F, Aubert D, Guys JM, Lottmann H, Lortat-Jacob S, Moscovici J, Mouriquand P, Ruffion A, Schmitt M: A multicentre study of the management of disorders of defecation in patients with spina bifida. Neurogastroenterol Motil 2006; 18(2): 123-8.

24-Isbister WH, Hubler M: Dynamic graciloplasty: a small and salutary experience. Ann Saudi Med 2000; 20(5-6): 390-3.

25-Tillin T, Chambers M, Feldman R: Outcomes of electrically stimulated gracilis neosphincter surgery. Health Technol Assess 2005; 9(28): iii, ix-xi, 1-102.

26-Gurusamy KS, Marzouk D, Benziger H: A review of contemporary surgical alternatives to permanent colostomy. Int $J$ Surg 2005; 3(3): 193-205.

27-O'Brien PE, Dixon JB, Skinner S, Laurie C, Khera A, Fonda D: A prospective, randomized, controlled clinical trial of placement of the artificial bowel sphincter (Acticon Neosphincter) for the control of fecal incontinence. Dis Colon Rectum 2004; 47(11): 1852-60.

28-Belyaev O, Muller C, Uhl W: Neosphincter surgery for fecal incontinence: a critical and unbiased review of the relevant literature. Surg Today 2006; 36(4): 295303.

29-Tomita R, Howard ER: Pathophysiological role of the cholinergic and adrenergic nerves in patients with allied disorders of Hirschsprung's disease (intestinal neuronal dysplasia and hypoganglionosis)--an in vitro experiment. Hepatogastroenterology 2007; 54(75): 725-8.

30-Tou JF, Li MJ, Guan T, Li JC, Zhu XK, Feng ZG: Mutation of RET proto-oncogene in Hirschsprung's disease and intestinal neuronal dysplasia. World J Gastroenterol 2006; 21; 12(7): 1136-9.

31-Shaul DB, Harrison EA, Muenchow SK: Avoidance of leakage and strictures when creating an invisible conduit for antegrade colonic enemas. J Pediatr Surg 2002; 37(12): 1768-71. 